\title{
Chronic Rhinosinusitis with Nasal Polyps appears to be A "Self-Limiting Disease" Lasting Approximately Eleven Years
}

Keywords: chronic rhinosinusitis; Nasal polyps; Sinus surgery; Age

\section{Abstract}

Not much is known about the influence of age and time on the results of sinus surgery (FESS) for chronic rhinosinusitis with nasal polyps (CRSWNP). We therefore used FESS as an objective sign of active disease and measured time between first and last surgical interventions in a follow-up of 10 years. We determined relation between age the total number of times of sinus surgery and age at time of the first operation ever and calculated the mean interval time between first and last operation. We found no relation between age and total number of surgical procedures. When we compared age at time of the first surgery to age at time of last surgery, we found a mean time interval of 11.1 years. We can conclude that the total number of sinus surgery procedures in patients' lifetimes seems to be independent of age. We think that CRSWNP is a self-limiting disease.

Chronic rhinosinusitis with nasal polyps (CRSwNP) is a chronic disease with a prevalence of $4-5 \%$ that results in high costs for society as a whole, mainly because of the need for repeated surgical interventions [1,2]. The prevalence of CRSwNP seems to increase with age, with the highest prevalence rate being seen in about the sixth decade of life $[3,4]$. We were not able to find any data about the natural course of the disease. We therefore used Sinus Surgery (FESS) as an objective sign of active disease and measured the time between the first and the last surgical interventions in a follow-up of 10 years.

In this study we sent a questionnaire to all adult patients who received FESS for CRSwNP in the AMC in Amsterdam between 2000 and 2005. We recorded their medical histories, including their complete sinonasal history with number of FESS procedures performed.

Questionnaires were returned by 151 out of 225 patients (103 men, 48 women; response rate $67 \%$ ). The mean total number of sinus surgery procedures during the patients' lifetimes was $3.4(\mathrm{SD} \pm 3.0)$. At the time of the questionnaire the mean time after the last surgery was 9.9 years $(\mathrm{SD} \pm 3.4)$.

When we compared the age at the time of the first surgery to the age at time of last surgery, we found a linear relationship with a mean time interval of 11.1 years ( $\mathrm{SD} \pm 11.1$, range 0 -52 years), a positive Pearson correlation of $\mathrm{r}=0.69(p=0.01)$ and a $95 \%$ Confidence Interval of 9.32-12.90. This means that there would seem to be standard active disease duration.

The table below shows the interval between the first and last procedures as compared with patient age at the time of the

\section{International Journal of}

\section{Otorhinolaryngology}

\section{Marjolein E. Cornet*, Sietze Reitsma, Gwijde F.J.P.M. Adriaensen and Wytske J. Fokkens}

Department of Otorhinolaryngology, Academic Medical Centre Amsterdam, Netherlands

\section{*Address for Correspondence}

Marjolein E Cornet, Department of Otorhinolaryngology, Academic Medical Centre Amsterdam, 1100 DD Amsterdam, Netherlands, E-mail: m.e.cornet@amc.nl

Submission: 29 September, 2016

Accepted: 18 October, 2016

Published: 24 October, 2016

Copyright: ๑ 2016 Cornet ME, et al. This is an open access article distributed under the Creative Commons Attribution License, which permits unrestricted use, distribution, and reproduction in any medium, provided the original work is properly cited.

questionnaire (Figure 1). We find no statistical differences between the age groups $(p=0.66)$.

Our study shows for the first time that the active disease duration based on the need for FESS in patients with CRSwNP is relatively constant at about 11 years, regardless of the age of onset. We hope this finding will hearten all CRSwNP patients requiring revision surgery and asking their doctors whether this awful disease will ever stop.

The limitations of our study were our decision to adopt the need for FESS as a sign of objective disease. Ideally, these results would be compared with nasal symptom scores and nasal endoscopy. Hopkins et al. showed recently that delayed surgical intervention for CRSwNP is associated with a higher need for post-operative health care than when patients undergo FESS in the first 12 months after diagnosis [5]. If we combine this finding with our results, we are justified in informing our patients that CRSwNP is a self-limiting disease but that early surgical intervention would seem to improve quality of life during the period when they are suffering from the disease.

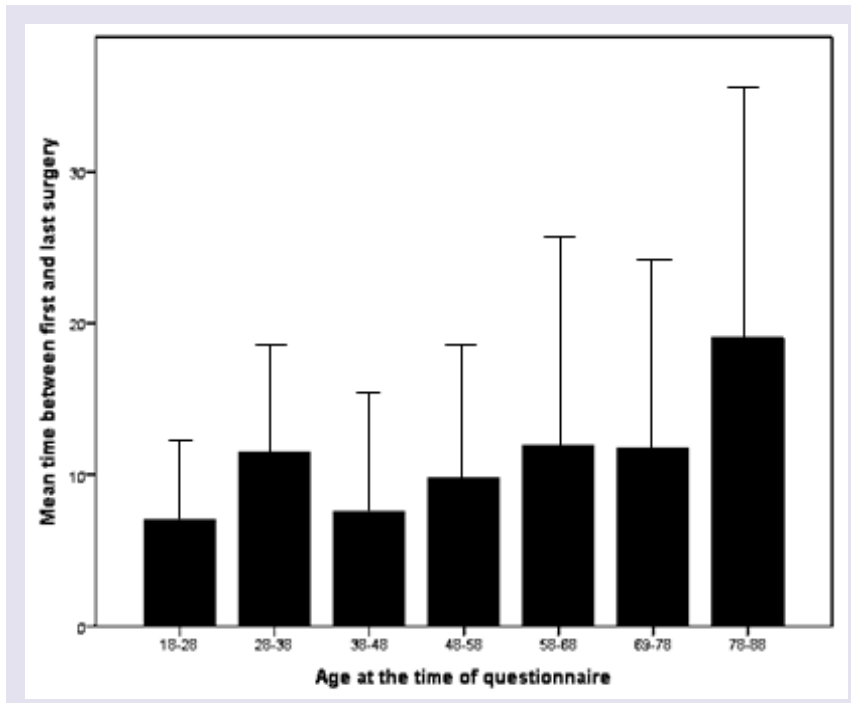

Figure 1: Interval between the first and last procedures as compared with patient age at the time of the questionnaire. 
Citation: Cornet ME, Reitsma S, Adriaensen GFJPM, Fokkens WJ. Chronic Rhinosinusitis with Nasal Polyps appears to be A "Self-Limiting Disease" Lasting Approximately Eleven Years. Inter J Otorhinolaryngology. 2016;3(1): 2.

\section{References}

1. Fokkens WJ, Lund VJ, Mullol J, Bachert C, Alobid I, et al. (2012) EPOS 2012 European position paper on rhinosinusitis and nasal polyps 2012. A summary for otorhinolaryngologists. Rhinology 50: 1-12.

2. Smith KA, Orlandi RR, Rudmik L (2015) Cost of adult chronic rhinosinusitis: A systematic review. Laryngoscope 125: 1547-1556.

3. We J, Lee WH, Tan KL, Wee JH, Rhee CS, et al. (2015) Prevalence of nasal polyps and its risk factors: Korean National Health and Nutrition Examination Survey 2009-2011. Am J Rhinol Allergy 29: e24-e28.

4. Johansson L, Akerlund A, Holmberg K, Melen I, Bende M (2003) Prevalence of nasal polyps in adults: the Skovde population-based study. Ann Otol Rhinol Laryngol 112: 625-629.

5. Hopkins C, Andrews P, Holy CE (2015) Does time to endoscopic sinus surgery impact outcomes in chronic rhinosinusitis? Retrospective analysis using the UK clinical practice research data. Rhinology 53: 18-24. 